\title{
Unraveling the Molecular Details of the Cell-ECM Interface: 3D Structures of Membrane-embedded Integrin Complexes
}

\author{
Xiao-Ping $\mathrm{Xu}^{1}$, Karen L Anderson ${ }^{1}$, Mark F Swift ${ }^{1}$, Niels Volkmann ${ }^{1}$ and Dorit Hanein ${ }^{1}$. \\ ${ }^{1 .}$ Bioinformatics and Structural Biology Program, Sanford-Burnham-Prebys Medical Discovery \\ Institute, La Jolla, California, United States
}

Adhesions between the cell and the extracellular matrix (ECM) are mechanosensitive multiprotein assemblies that transmit force across the cell membrane and regulate biochemical signals in response to changes in the mechanical environment. These combined functions in force transmission, signaling and mechanosensing are crucial for cell behaviors in development, homeostasis and disease. Here I will focus on our studies of integrin-mediated adhesions. Despite advances in our understanding of the protein composition, interactions and regulation of these large multiprotein assemblies, our understanding of how forces affect the dynamic organization of adhesions and how they induce specific signaling events remains limited. Insights across multiple structural levels are acutely needed to establish the molecular bases of mechanotransduction.

If cells respond to forces through distinct structural adaptations with talin and integrin forming the major axis to connect the extracellular matrix with the actin cytoskeleton, defining how the actin-binding protein, talin and its extracellular receptors integrin interactions govern adhesions' ultrastructure, will allow us to unravel the basis force transfer. Integrins are bidirectional, allosteric transmembrane receptors expressed on the cell surface and are believed to require an activation step that triggers extracellular ligand binding and initiates adhesion. We developed a system for imaging such membrane receptors in small lipid-bilayer islands, nanodiscs that allow application of single-particle analysis of membrane-embedded particles [1]. This system serves as a basis for investigation of integrin-based complexes in reconstituted systems. Using cryo-electron microscopy, multireference single-particle reconstruction methods, and statistics-based computational fitting approaches, we determined threedimensional structures of human integrin allbb3 embedded in a lipid bilayer using this nanodisc technology [2], while bound to domains of the cytosolic regulator talin and to extracellular ligands. We also determined the conformations of integrin in solution by itself to localize the membrane and the talin-binding site.

Our study shows that aIIbb3 integrins and integrins embedded in nanodiscs exist in an equilibrium of states even while they are bound to cytosolic and extracellular ligands. These conformations range from a compact nodular structure similar to the crystal structures of ectodomains to a fully upright conformation and two intermediate states. The addition of talin head domains, RGD peptide, and phospholipid bilayer to the system significantly shifts the conformational equilibrium toward the upright conformation, suggesting that integrin activation occurs by equilibrium shifts and not a simple transition from a bent to extended conformation. The nanodisc technology opens up the avenue to detailed structural investigation of the integrin inside-out and outside-in activation mechanisms and the role of additional integrin binding partners such as talin. Templates of these integrin conformations are invaluable in our in silico identification of integrins in cellular environments, our ultimate goal. Additional efforts in the lab we directed into defining the structures of vinculin isoforms [3], vinculin, talin, and actin-talin complexes [4]. These define a basic library of motifs for template-based searches in 
cellular tomograms to characterize adhesion sites [5] and for fitting of high-resolution structures into the lower resolution cellular tomograms.

Next, to identify these assemblies and their nanoscale three-dimensional architecture in intact mammalian cells, we employed correlative light microscopy guided by designated fluorescence in conjunction with electron cryo-tomography. These correlative approaches, once with image analysis, data mining allows to directly view in 3D while in cellular environment, the molecular architecture of single molecular components of these fascinating mechano-sensing nanomachines. In the past, ultrastructural studies of such mutliprotein networks were based on the analysis of small numbers of tomograms or platinum replicas in 2D. Recent advances in cryoEM hardware, and image analysis capabilities revolutionize the cryoET field by enabling the generation of high-resolution structural data, in a robust and reproducible fashion. Here taking advantage of the Titan Krios microscope equipped with Direct Detector and Volta Phase Plate at SBP we analyzed a total of several hundred cryotomograms using highly efficient automated actin tracing protocols [6,7] to determine composition and the nano-scale organization of these networks aiming for defining single integrin ECM connections in situ [8].

\section{References:}

[1] Xu XP et al, Cell Death Dis. 4 (2013), e683.

[2] Xu XP et al, Biophys J. 110 (2016), p. 798.

[3] Janssen ME et al, J Cell Biol. 197 (2012), p. 585.

[4] Goult BT, Xu XP, Gingras AR, Swift M, Patel B, Bate N, Kopp PM, Barsukov IL, Critchley DR,

[5] Volkmann N, Hanein D. J Struct Biol. 184 (2013), p. 21. PMC3799832

[6] Page C, Hanein D, Volkmann N. Ultramicroscopy. 155 (2015), p. 20.

[7] Xu XP and Volkmann N, Arch Biochem Biophys. 581 (2015), p. 49.

[8] This work was supported by NIH program project grant P01 GM098412 and R01CA179087(DH, NV). NIH grants S10 OD012372(DH) and P01 GM098412-S1(DH) funded the purchase of the Titan Krios TEM and Falcon II direct detection imaging device. 